“ (C) 2018 IEEE. Personal use of this material is permitted. Permission from IEEE must be obtained for all other uses, in any current or future media, including

reprinting/republishing this material for advertising or promotional purposes, creating new collective works, for resale or redistribution to servers or lists, or reuse of any copyrighted component of this work in other works." 


\section{A Hybrid Feedforward-feedback Hysteresis Compensator in Piezoelectric Actuators Based on Least Squares Support Vector Machine}

\begin{abstract}
Hysteresis nonlinearity of piezoelectric actuators degrades the positioning accuracy of micro-/nano-positioning systems. To overcome this problem, an innovative hysteresis compensator based on least squares support vector machine (LSSVM) is proposed in this paper. First, the LSSVM hysteresis modeling is presented using Nonlinear Auto Regressive eXogenous (NARX) structure. To compensate for the hysteresis behavior, two feedforward control schemes according to different inputs of NARX model are proposed and analyzed separately. Then, a hybrid feedforward controller combining both the control schemes is put forward to revise the model input. To further improve the tracking performance, the hybrid feedforward control combined with the feedback control is realized. The comparative study reveals the superior tracking performance of feedforward-feedback control scheme over hybrid feedforward control or feedback control. Moreover, the hybrid feedforward-feedback control scheme is capable of tracking different testing waveforms with negligible errors, which confirms the effectiveness and generalization ability of the proposed approach.
\end{abstract}

Index Terms-Feedforward-feedback, hysteresis compensation, piezoelectric actuator.

\section{INTRODUCTION}

$\mathrm{P}$ iezoelectric actuators (PZAs) are widely used in micro-/nano-positioning systems due to the merits of small size, high positioning resolution, rapid response speed, and large driving force Error! Reference source not found., [2]. However, the inherent hysteresis of PZA can produce an open-loop positioning error as much as $10-15 \%$ of the motion range [3]. Therefore, the hysteresis of PZAs should be suppressed to improve the positioning precision and dynamic performance.

Although electrical charge control can attenuate the hysteresis using linear relationship between the charge and displacement [4], [5], it is not practical due to the complexity of the implementation and the reduction in the operating range. Thus, the voltage driving control is widely adopted and the hysteresis modeling is the key point of this method.

Since the characteristic of the piezoelectric hysteresis depends not only on the amplitude but also on the frequency of input voltage signals, traditional rate-independent hysteresis models could yield errors subject to dynamic inputs with different frequencies [6]-[12]. To characterize the rate-dependent hysteresis, some models were put forward, such as the improved Preisach model [13], [14], improved Prandtl-Ishlinskii model [15], [16], and time series similarity model [17]-[19]. However, these models have a lot of parameters to be determined, which complicate the modeling process. In contrast, the support vector machine (SVM), based on statistical theory and structural risk minimization principle [20], outperforms the artificial neural network (ANN) in terms of global optimization and generalization capability [21]-[23] and it shows good performance in hysteresis modeling [24], [25]. As an extension of SVM, the least squares support vector machine (LSSVM) overcomes the defect of slow training speed in SVM by solving a linear equation set rather than a quadratic optimization problem [26]. Also, LSSVM has fewer parameters to be tuned [27], which means it can achieve accurate regression more easily. The hyperparameters in LSSVM are usually optimized by intelligent optimization algorithms to improve the regression accuracy [28]-[30]. Particle swarm optimization (PSO), as one of intelligent optimization algorithms, is widely used in the field of parameters optimization because of its easy operation and excellent convergence ability [31], [32].

The feedforward control method with an inverse hysteresis model is an effective way for hysteresis compensation [33]-[35]. However, LSSVM can only model one-to-one mapping, whereas the hysteresis nonlinearity is a multivalued mapping. A practical way is to employ the Nonlinear Auto Regressive eXogenous (NARX) model, which represents an input-output recursive model [36]. NARX model is widely employed for nonlinear system identification [37]-[39], where the current output is predicted by the current and previous inputs and previous outputs. For the nonlinear system control, however, the previous desired outputs and the previous measured outputs can both be taken as the input of the NARX model. Some literatures take the previous desired outputs into the NARX model [25], [40], whereas it still needs more research to determine the optimal structure of the feedforward controller.

In this paper, two feedforward control schemes based on different sources of NARX model inputs are proposed and analyzed separately. Then, a hybrid feedforward control scheme combining these two methods in a certain ratio is developed. And the ratio selection is discussed to achieve the 
optimal positioning accuracy. To further suppress the tracking errors, the combination of hybrid feedforward control with feedback control is adopted. Finally, to assess the performance of the proposed control schemes, control experiments of a PZA are undertaken. The explicit comparative studies are conducted with the traditional PID feedback control to validate the effectiveness of the proposed compensators.

The rest of this paper is organized as follows. Section II provides a brief review of LSSVM and describes the hyperparameters optimization procedure based on PSO. Section III presents the experiment results for hysteresis modeling. In section $\mathrm{IV}$, controllers are proposed and verified for hysteresis compensation. Conclusions are finally provided in section $\mathrm{V}$.

\section{LSSVM AND PARAMETER OPTIMIZATION}

\section{A. LSSVM for Hysteresis Modeling}

In order to convert the hysteresis multivalued mapping into an one-to-one mapping, the nonlinear regression model is established based on NARX model. That is

$$
y_{k}=f\left(\boldsymbol{x}_{k}\right)+\xi_{k}
$$

with

$$
\boldsymbol{x}_{k}=\left[\begin{array}{lllllll}
u_{k} & u_{k-1} & \cdots & u_{k-n} & y_{k-1} & \cdots & y_{k-m}
\end{array}\right]
$$

where $u_{k}$ and $y_{k}$ denote the input voltage and output displacement of the system at time instance $k, \xi_{k}$ is the prediction error, $f(\cdot)$ represents the nonlinear regression model, and $m$ and $n$ define the system orders. It is found that as the system orders increase, the training error gradually decreases, while the testing error first decreases and then increases [25] and the computational cost increases. To make a compromise, $m$ and $n$ are both set to 3 .

The LSSVM is employed to model the piezoelectric hysteresis and the model $f(\cdot)$ takes the form

$$
y(\boldsymbol{x})=\boldsymbol{\omega}^{\mathrm{T}} \varphi(\boldsymbol{x})+b
$$

where a nonlinear function $\varphi(x)$ maps the input space into a high-dimensional space. $\left\{\boldsymbol{x}_{k}, y_{k}\right\}_{k=1}^{N}$ is given as the training set, where $N$ is the sample size. $\omega$ and $b$ are the parameters which can be determined by solving the following optimization problem

$$
\begin{aligned}
& \min _{\boldsymbol{\omega}, \xi, b} J(\boldsymbol{\omega}, \xi)=\frac{1}{2} \boldsymbol{\omega}^{\mathrm{T}} \boldsymbol{\omega}+\frac{1}{2} C \sum_{k=1}^{N} \xi_{k}^{2} \\
& \text { s.t. } y_{k}=\boldsymbol{\omega}^{\mathrm{T}} \varphi\left(\boldsymbol{x}_{k}\right)+b+\xi_{k}
\end{aligned}
$$

where $C$ represents the regularization factor which balances the training error and model complexity. The Lagrangian function of problem (4) is then expressed as

$$
\begin{aligned}
L(\boldsymbol{\omega}, b, \xi, \alpha) & =J(\boldsymbol{\omega}, \xi) \\
& -\sum_{k=1}^{N} \alpha_{k}\left[\boldsymbol{\omega}^{\mathrm{T}} \varphi\left(\boldsymbol{x}_{k}\right)+b+\xi_{k}-y_{k}\right]
\end{aligned}
$$

where $\alpha_{k}$ are the Lagrangian multipliers. The optimal solutions meet the following conditions

$$
\left\{\begin{array}{l}
\frac{\partial L}{\partial \omega}=0 \rightarrow \omega=\sum_{k=1}^{N} \alpha_{k} \varphi\left(\boldsymbol{x}_{k}\right) \\
\frac{\partial L}{\partial \xi_{k}}=0 \rightarrow \alpha_{k}=C \xi_{k} \\
\frac{\partial L}{\partial b}=0 \rightarrow \sum_{k=1}^{N} \alpha_{k}=0 \\
\frac{\partial L}{\partial \alpha_{k}}=0 \rightarrow \omega^{\mathrm{T}} \varphi\left(\boldsymbol{x}_{k}\right)+b+\xi_{k}-y_{k}=0 .
\end{array}\right.
$$

Eliminating $\omega$ and $\xi$, the solutions are given by the following linear equations

$$
\left[\begin{array}{cc}
0 & \boldsymbol{e}_{N \times 1}^{\mathrm{T}} \\
\boldsymbol{e}_{N \times 1} & \boldsymbol{\Omega}+\boldsymbol{I}_{N} / C
\end{array}\right]\left[\begin{array}{l}
b \\
\boldsymbol{\alpha}
\end{array}\right]=\left[\begin{array}{c}
0 \\
\boldsymbol{Y}
\end{array}\right]
$$

where $\quad \boldsymbol{e}_{N \times 1}=[1 ; 1 ; \cdots ; 1] \quad, \quad \boldsymbol{\alpha}=\left[\alpha_{1} ; \alpha_{2} ; \ldots ; \alpha_{N}\right]$, $\boldsymbol{Y}=\left[y_{1} ; y_{2} ; \ldots ; y_{N}\right] \quad, \quad \boldsymbol{I}_{N}$ is an identity matrix, $\Omega_{i j}=\varphi^{\mathrm{T}}\left(\boldsymbol{x}_{i}\right) \cdot \varphi\left(\boldsymbol{x}_{j}\right)=K\left(\boldsymbol{x}_{i}, \boldsymbol{x}_{j}\right) . K$ is the kernel function and the radial basis function kernel is used in this paper

$$
K\left(\boldsymbol{x}_{i}, \boldsymbol{x}_{j}\right)=\exp \left(-\left\|\boldsymbol{x}_{i}-\boldsymbol{x}_{j}\right\|^{2} / 2 \sigma^{2}\right)
$$

where $\sigma$ is the kernel width parameter.

After obtaining $b$ and $\alpha$ from (7), the regression model of LSSVM becomes

$$
y(\boldsymbol{x})=\sum_{k=1}^{N} \alpha_{k} K\left(\boldsymbol{x}_{k}, \boldsymbol{x}\right)+b .
$$

\section{B. Hyperparameters Optimization Based on PSO}

The selection of hyperparameters $C$ and $\sigma$ is significant for achieving an accurate LSSVM model. In this section, PSO is adopted to optimize the hyperparameters due to its fast convergence and robustness.

PSO algorithm simulates the birds flock's behavior of preying on food and searching for the optimal position. PSO consists of a swarm of interacting particles searching in an $L$-dimensional search space of the problem's solutions ( $L$ is the size of hyperparameters). Each particle can be described by its current position and velocity. For instance, the position and velocity of particle $i$ at iteration $t$ can be expressed as $\boldsymbol{p}_{i}^{t}=\left\{p_{i 1}^{t}, p_{i 2}^{t}, \cdots p_{i L}^{t}\right\}$ and $\boldsymbol{v}_{i}^{t}=\left\{v_{i 1}^{t}, v_{i 2}^{t}, \cdots v_{i L}^{t}\right\}$. Each particle updates its speed and location by tracking individual best known position pbest $_{i}$ and swarm's best position gbest. The velocity and position of particle $i$ are updated according to the following two formulas

$$
\begin{gathered}
\boldsymbol{v}_{i}^{t+1}=\eta \boldsymbol{v}_{i}^{t}+c_{1} \times r_{1} \times\left(\boldsymbol{p b e s t}_{i}-\boldsymbol{p}_{i}^{t}\right)+c_{2} \times r_{2} \times\left(\text { gbest }-\boldsymbol{p}_{i}^{t}\right) \\
\boldsymbol{p}_{i}^{t+1}=\boldsymbol{p}_{i}^{t}+\boldsymbol{v}_{i}^{t+1}
\end{gathered}
$$

where $\eta$ denotes the inertia weight, $c_{1}$ and $c_{2}$ are the learning factors, and $r_{1}$ and $r_{2}$ are random numbers between 0 and 1 .

The performance of each particle is evaluated by the prediction error using cross-validation. That is

$$
f(C, \sigma)=\frac{1}{N_{\text {test }}} \sum_{k=1}^{N_{\text {test }}}\left(y_{k}-\hat{y}_{k}\right)^{2}
$$


where $y_{k}$ and $\hat{y}_{k}$ are the $k$ th actual output and predicting output, and $N_{\text {test }}$ is the size of test samples.

Thus, the algorithm steps of hyperparameters selection using PSO are provided as follows:

1) Establish the PSO with a group of particles with random positions and velocities.

2) For each particle $i$, build the regression model and predict the outputs for test samples, and evaluate the particle's performance using (10).

3) Replace pbest $_{i}$ with the particle $i$ if the latter is superior. Replace gbest with the best particle of the population if the latter is superior.

4) Update the velocity and position of each particle based on (9).

5) Repeat steps 2-4 until the stop criteria are satisfied.

\section{EXPERIMENTS FOR HYSTERESIS MODELING}

\section{A. Experimental Setup}

The experiments are carried out on a piezoelectric actuator MPT-1JRL002 (withstand-voltage range: -30 to $150 \mathrm{~V}$ and displacement resolution: $0.01 \mu \mathrm{m}$ ). Fig. 1 shows the system devices. The hardware-in-the-loop simulation system produces an analogy voltage output, which is then amplified by a power amplifier to drive the PZA. The output displacement of PZA is measured by a resistance strain gauge sensor, which is installed within the PZA as a micrometer and then transmitted back to the hardware-in-the-loop simulation system.

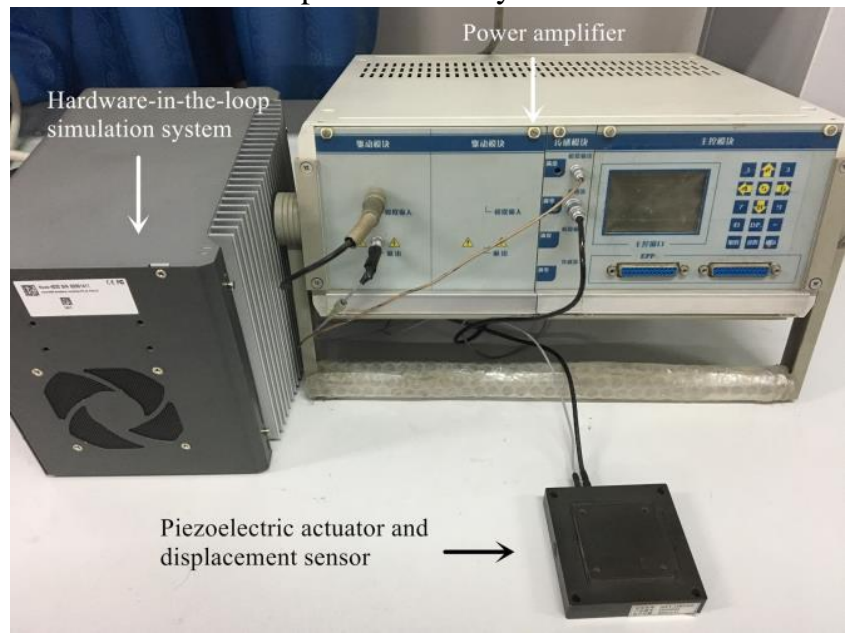

Fig. 1. Piezoelectric actuator experiment devices.

\section{B. LSSVM Model Training and Testing}

Considering the rate-dependent behavior of piezoelectric hysteresis, the training data must excite as many states of piezoelectric actuator as possible. Thus, the random sinusoidal input voltage shown in Fig. 2(a) is used for training and the corresponding output displacement is shown in Fig. 2(b).

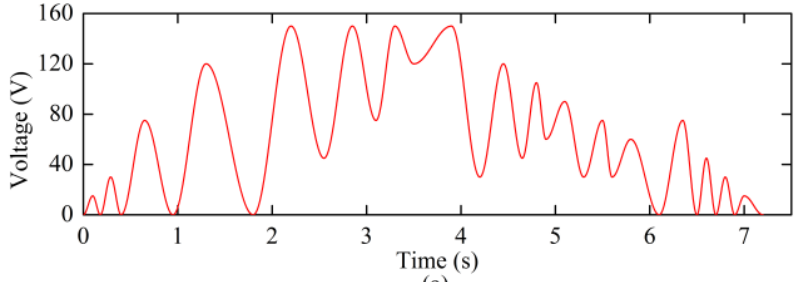

(a)

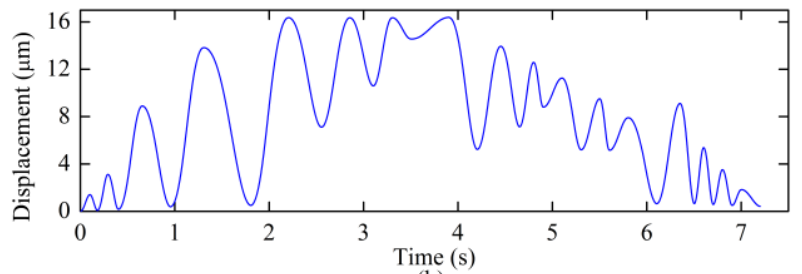

(b)

Fig. 2. Training data set: (a) input voltage, (b) output displacement.

Given the training data set, the hyperparameters are set as $C$ $=1.38 \times 10^{5}$ and $\sigma=1.76$ by PSO. Then two sets of random input waveforms are employed to test the performance of the regression model. As described in Fig. 3, the results show that the proposed algorithm can achieve accurate regression for the PZA hysteresis under random input excitation.

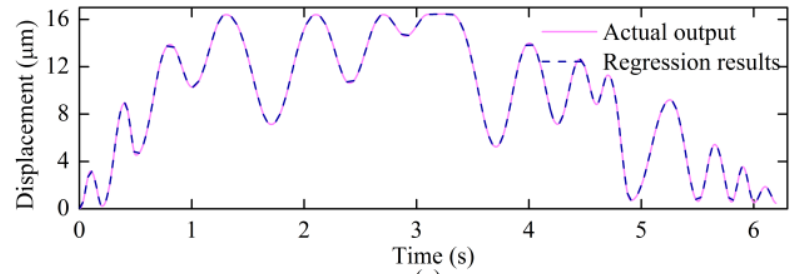

(a)

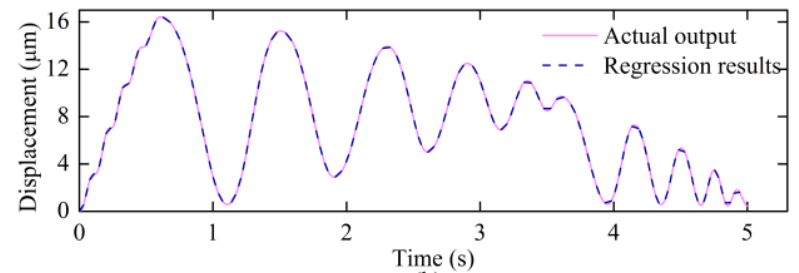

(b)

Fig. 3. Hysteresis modeling results under random input excitation: (a) test input 1 , (b) test input 2 .

Furthermore, the root mean squared error (RMSE) is employed to evaluate the accuracy of regression model, which is expressed as

$$
R M S E=\sqrt{\frac{1}{n} \sum_{k=1}^{n}\left(y_{k}-\hat{y}_{k}\right)^{2}}
$$

where $y_{k}$ and $\hat{y}_{k}$ are true value and predicted value respectively.

The regression model produces RMSEs of $0.00504 \mu \mathrm{m}$ and $0.00543 \mu \mathrm{m}$ respectively for the two testing samples, accounting for $0.0315 \%$ and $0.0339 \%$ of the motion range. By comparison, the Preisach model produces RMSEs of $3.98 \%$ and $3.64 \%$ as shown in Fig. 4. Therefore, it can be concluded that the LSSVM can identify the hysteresis nonlinearity of piezoelectric actuators far more accurate than the traditional Preisach model. 


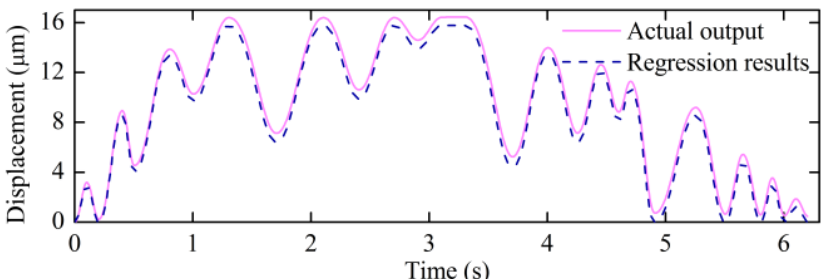

(a)

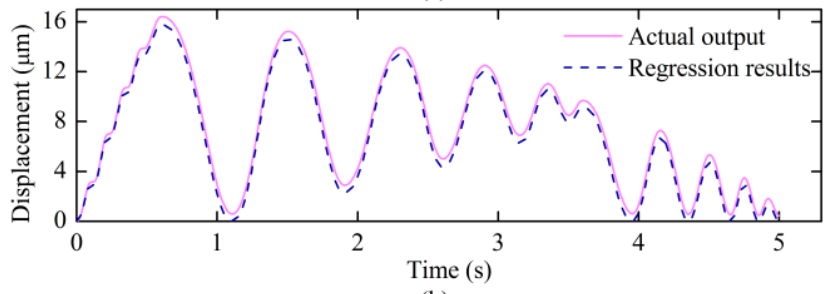

(b)

Fig. 4. Hysteresis modeling results of Preisach model under random input excitation: (a) test input 1, (b) test input 2 .

\section{HYSTERESIS COMPENSATION}

In this section, controllers are designed and discussed based on LSSVM hysteresis inverse model to compensate the hysteresis nonlinearity. Experiment results verify the effectiveness of the proposed control scheme.

\section{A. Feedforward Controller}

Although the hysteresis phenomenon limits the positioning accuracy of piezoelectric actuators, the feedforward control method with an inverse hysteresis model is an effective way for hysteresis compensation.

To build the LSSVM inverse model, a similar method as shown in section II can be taken by selecting the current and previous output displacements and previous input voltage as exogenous inputs to predict the current input voltage. The hysteresis inverse model can be expressed as

$$
u_{k}=f\left(\boldsymbol{x}_{k}\right)+\xi_{k}
$$

with

$$
\boldsymbol{x}_{k}=\left[\begin{array}{lllllll}
y_{k} & y_{k-1} & \ldots & y_{k-n} & u_{k-1} & \ldots & u_{k-m}
\end{array}\right]
$$

where $m$ and $n$ are both set to 3 .

Once trained offline, the hysteresis inverse model is utilized for online feedforward control. As for the control scheme, the previous desired outputs and the previous measured outputs can both be taken as the previous displacements of input into the hysteresis inverse model. Therefore, according to different source of the model input, two different feedforward control schemes are proposed and studied. The control diagrams are shown in Fig. 5 and Fig. 6. The first method appears like a feedback controller but the measured displacement is only employed for inverse model calculation; the second method is actually an open-loop controller.

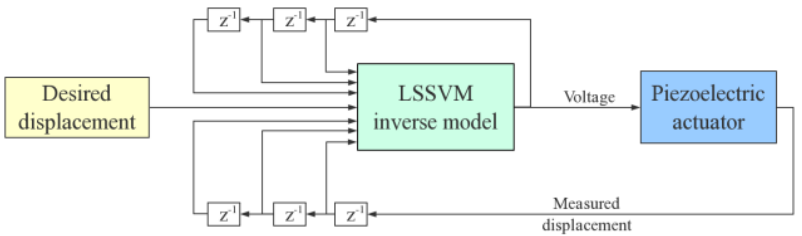

Fig. 5. Block diagram of feedforward control method 1.

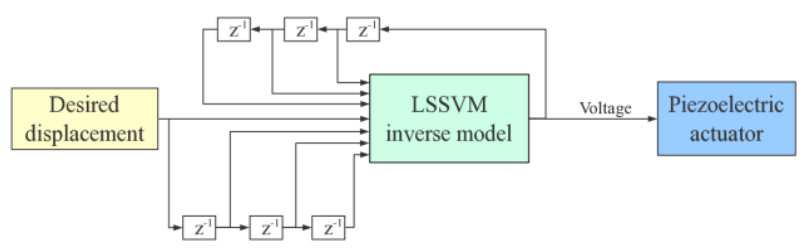

Fig. 6. Block diagram of feedforward control method 2.

The small amount of noise in the output displacement could affect the accuracy of inverse model. Thus, the training data is smoothed first using moving average before model training. Two sets of test samples were employed to analyze the performance of two control methods. As shown in Fig. 7, the test results reveal that the output displacement does not track the given displacement well for the first method. The output oscillates intensely and deviates from the desired displacement with RMSEs of $3.49 \mu \mathrm{m}$ and $2.42 \mu \mathrm{m}$. It is due to the fact that the previous displacement and the current displacement are close to each other in the training sample. If the real-time output deviates from the given outputs, however, the input state of the inverse model is not reflected in the training samples, and therefore the output displacement deviates from the expected output. For the second method, the output displacement can roughly track the given displacement with RMSEs of $0.49 \mu \mathrm{m}$ and $0.37 \mu \mathrm{m}$, because the inverse modeling error in the output of the inverse model is transferred to the input side, resulting in a large deviation in the model output.
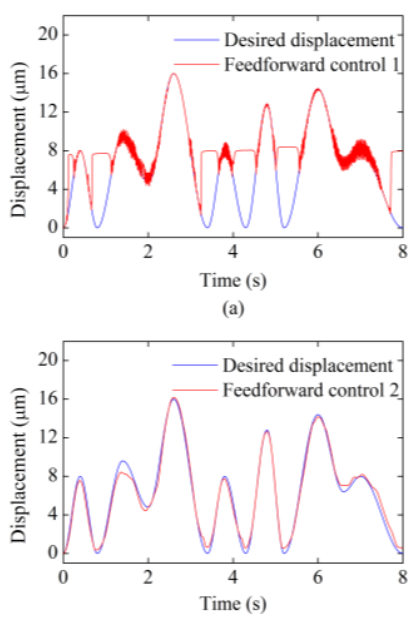

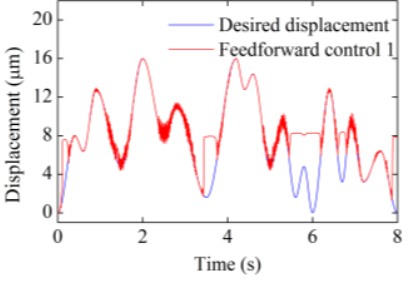

(b)

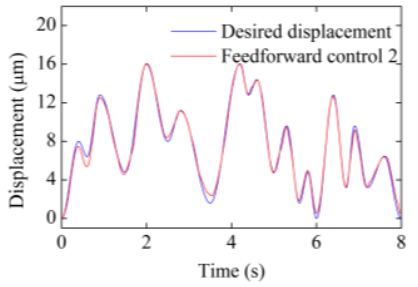

Fig. 7. (a) and (b) Tracking results of the first control schemes tested by two testing samples. (c) and (d) Tracking results of the second control schemes tested by two testing samples.

\section{B. Hybrid Feedforward Controller}

It seems that the open-loop controller (i.e. the second method) has the relatively superior performance. To revise the 
previous displacement input of the model for the open-loop controller, some measured displacement is added, which can be considered as a correction. Thus, a hybrid feedforward control scheme that combines the two methods are proposed, where the previous displacement input is composed of the measured displacement and the desired displacement in a certain ratio. The control block diagram is shown in Fig. 8, in which $p$ is the ratio of the desired displacement. Specifically, it represents the feedforward control method 1 when $p=0$ and the feedforward control method 2 when $p=1$.

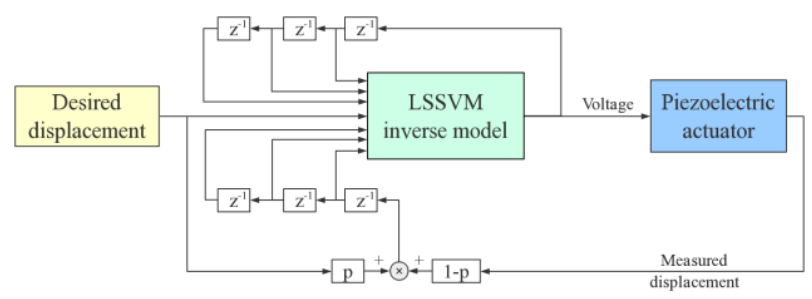

Fig. 8. Block diagram of hybrid feedforward control scheme.

The selection of the parameter $p$ has a great influence on the accuracy of the system. To select the appropriate value, $p$ is evenly divided between 0 and 1 and the system performance is analyzed by two sets of test data. The testing errors are depicted in Fig. 9. It is found that the system performs the best when $p=$ 0.7 and the RMSEs for two data sets are $0.05214 \mu \mathrm{m}$ and $0.03186 \mu \mathrm{m}$, accounting for $0.326 \%$ and $0.199 \%$ of the motion range respectively. Therefore, $p$ is set to 0.7 and the corresponding tracking results are shown in Fig. 10. It is observed that the output displacement can track the desired displacement accurately, except for the non-negligible deviations at extreme points.

The performance of the hybrid feedforward controller is far superior to those of the two feedforward controllers when $p$ is around 0.7. In general, the optimal value of $\mathrm{p}$ should be determined with enumeration method for different actuators or different model parameters.

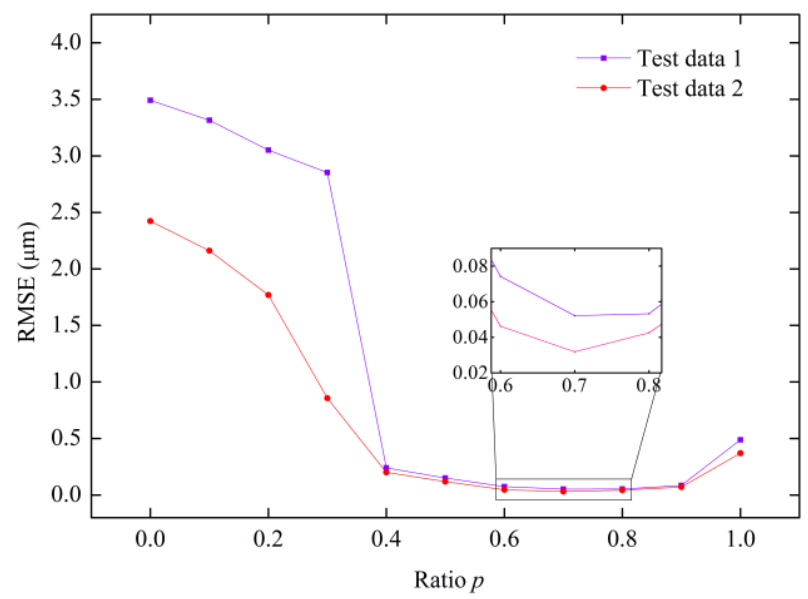

Fig. 9. Effects of $p$ value on the performance of the hybrid feedforward control system.

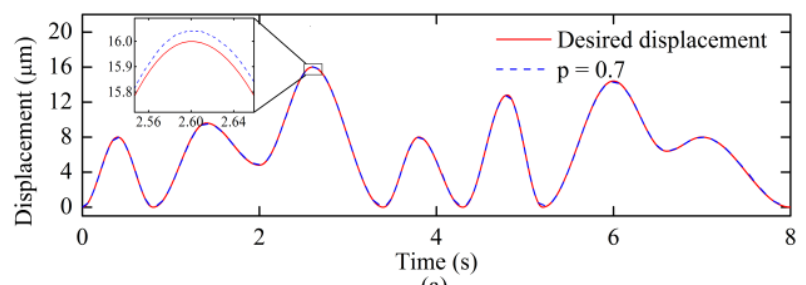

(a)

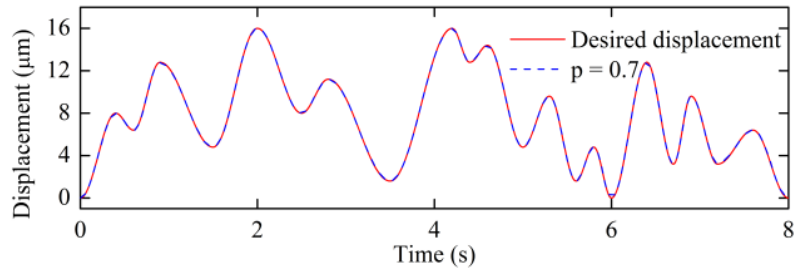

(b)

Fig. 10. Tracking results of the hybrid feedforward control system when $p=$ 0.7: (a) test data 1, (b) test data 2.

\section{C.Hybrid Feedforward-Feedback Controller}

To further improve the tracking accuracy, the hybrid feedforward control combined with the feedback control strategy is realized. Fig. 11 shows the control scheme. The incremental PID algorithm is employed as the feedback controller, and the input signal of piezoelectric actuator can be expressed as follows

$$
\begin{aligned}
v_{c}(k) & =v_{f}(k)+v_{b}(k-1)+K_{p}[e(k)-e(k-1)]+K_{i} e(k) \\
& +K_{d}[e(k)-2 e(k-1)+e(k-2)]
\end{aligned}
$$

where $v_{c}$ represents the control voltage, $v_{f}$ is the model output voltage, $v_{b}$ is the feedback control voltage, $e$ denotes the tracking error, and $K_{p}, K_{i}$ and $K_{d}$ are PID controller parameters.

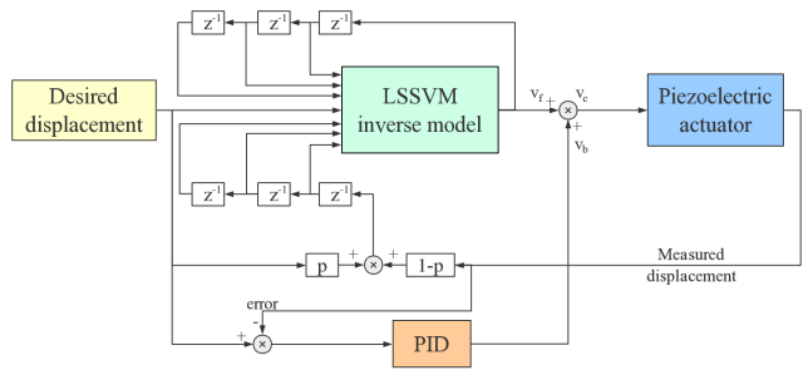

Fig. 11. Block diagram of the hybrid feedforward-feedback controller. 


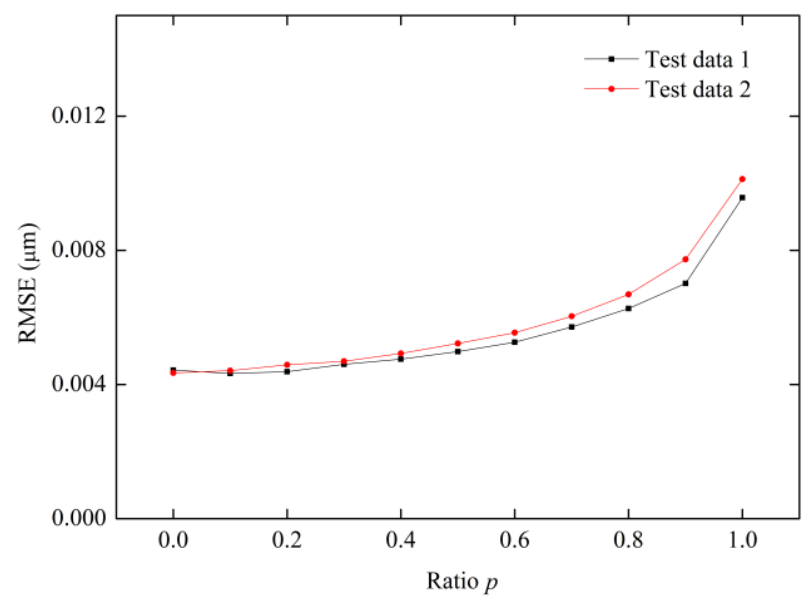

Fig. 12. The effects of $p$ value on the performance of the hybrid feedforward-feedback controller.

Two data sets are employed to test the effect of $p$ value on the model performance. As shown in Fig. 12, the test results show that the tracking error rises slowly with the increase of $p$, although the ascending trend is not obvious. RMSEs of the two data sets range from $0.004 \mu \mathrm{m}$ up to $0.005 \mu \mathrm{m}$ when $p$ increases from 0 to 0.5 . Specifically, the tracking performance of the system is optimal when $p=0$, which means that the previous displacement of the input into the hysteresis inverse model comes entirely from the actual output. The optimal RMSEs of the two test samples are $0.00434 \mu \mathrm{m}$ and $0.00443 \mu \mathrm{m}$ respectively, accounting for $0.0277 \%$ and $0.0271 \%$ of the motion range. The tracking results of the control system are shown in Fig. 13.

The introduction of the feedback controller makes the feedforward method 1 more accurate since the inverse model is flexible to output the required voltage with fast response, while the output of the inverse model for method 2 produces fixed error. Thus, the controller gradually transmits from the method 1 to method 2 with the increase of $\mathrm{p}$ from 0 to 1 and the corresponding RMSE rises slowly as shown in Fig. 12.

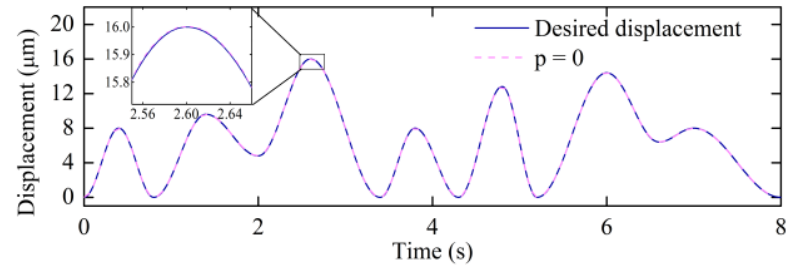

(a)

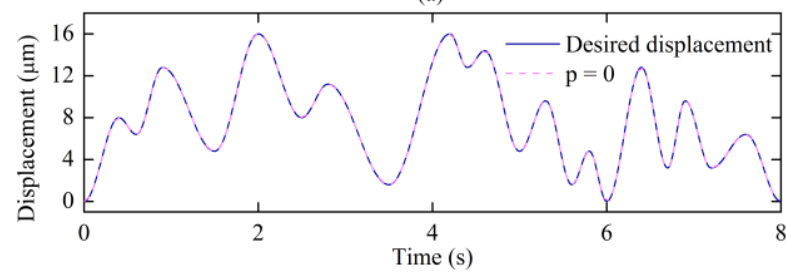

(b)

Fig. 13. Tracking results of the hybrid feedforward-feedback controller when $p=0$ : (a) test data 1 , (b) test data 2 .

\section{D.Controller comparison and generalization}

For better comparison, the tracking results of PID feedback controller and Preisach feedforward controller are shown in
Fig. 14 and Fig. 15. The tracking RMSEs of different control scheme are summarized in TABLE I. The Preisach feedforward controller yields the worst RMSEs of $2.4 \%$ and $2.24 \%$. The hybrid feedforward control produces RMSEs of $0.326 \%$ and $0.199 \%$ for two data sets, which are slightly improved by $21.6 \%$ and $43.1 \%$ as compared with the feedback control. Furthermore, the hybrid feedforward-feedback control enhances the tracking accuracy by more than 11 times and 7 times in comparison with the hybrid feedforward control.

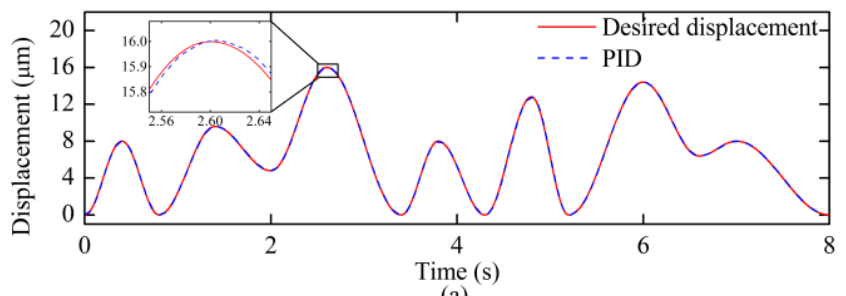

(a)

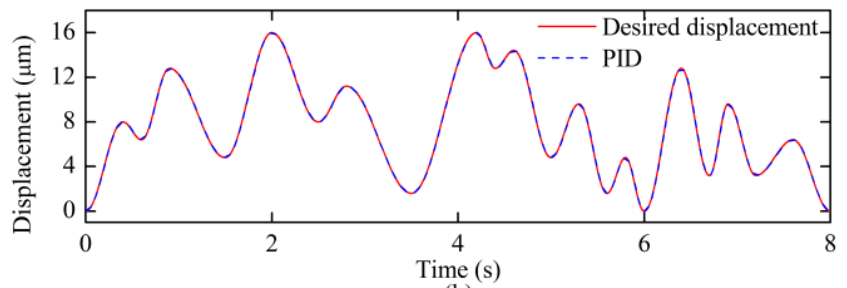

(b)

Fig. 14. Tracking results of the PID feedback controller: (a) test data 1, (b) test data 2 .

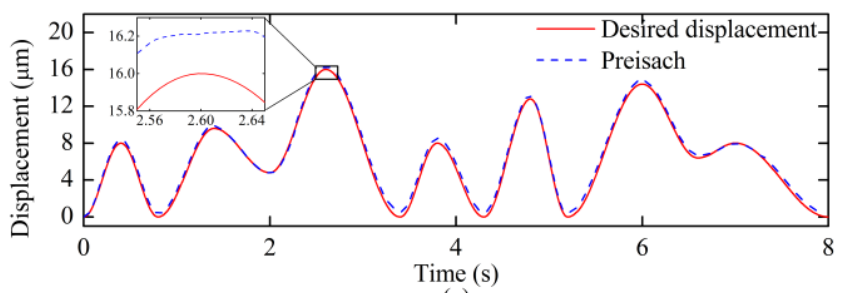

(a)

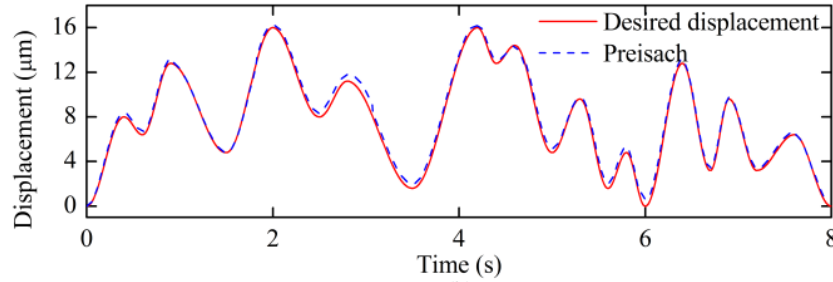

(b)

Fig. 15. Tracking results of the Preisach feedforward controller: (a) test data 1 , (b) test data 2.

TABLE I

TRACKING RMSES (\%) OF DIFFERENT CONTROL SCHEMES TESTED BY TWO SAMPLES.

\begin{tabular}{lcccc}
\hline \hline Data set & $\begin{array}{c}\text { Preisach } \\
\text { feedforward }\end{array}$ & $\begin{array}{c}\text { PID } \\
\text { Feedback }\end{array}$ & $\begin{array}{c}\text { Hybrid } \\
\text { Feedforward } \\
(p=0.7)\end{array}$ & $\begin{array}{c}\text { Feedforward- } \\
\text { feedback }(p= \\
0)\end{array}$ \\
\hline Test data 1 & 2.40 & 0.416 & 0.326 & 0.0277 \\
Test data 2 & 2.24 & 0.350 & 0.199 & 0.0271 \\
\hline \hline
\end{tabular}

A good generalization capacity of the hybrid feedforward-feedback controller is evident from the experimental results shown in Fig. 16. It is found that the 
controller is able to track waveforms quite different from the training data accurately, such as the random triangular wave and the trapezoidal wave, with RMSEs of $0.0631 \%$ and $0.0632 \%$ respectively.

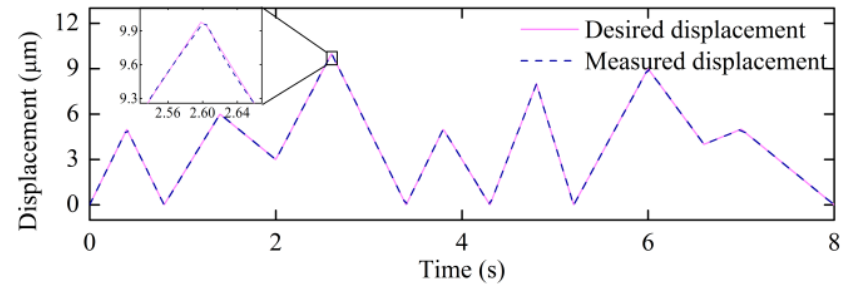

(a)

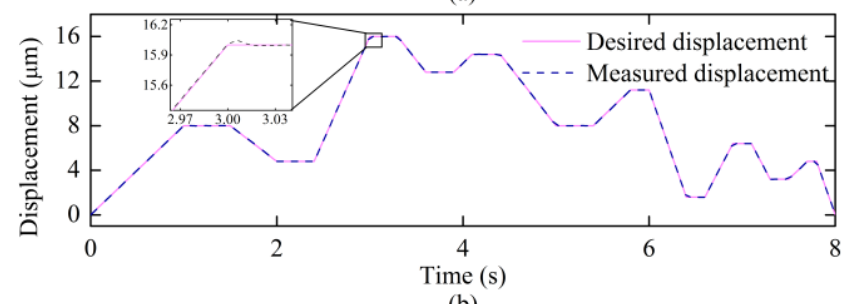

(b)

Fig. 16. Tracking performance of the hybrid feedforward-feedback controller for different waveforms: (a) triangular wave, (b) trapezoidal wave.

\section{CONCLUSION}

In this paper, the NARX-based LSSVM is demonstrated to be effective to model and compensate for the hysteresis of piezoelectric actuators. The hybrid feedforward controller combining the desired outputs and the measured outputs as the input of NARX model is first proposed for hysteresis compensation, where the tracking performance heavily depends on the combination ratio. It is found that the controller performs the best when the desired outputs account for $70 \%$. The experimental results reveal the superior performance of the hybrid feedforward controller over the PID feedback controller. To further improve the tracking performance, the hybrid feedforward control combined with the feedback control is realized, which outperforms the stand-alone feedback or feedforward controller significantly. The optimal performance of the feedforward-feedback controller can be obtained when the input of NARX model comes entirely from the actual outputs. Moreover, due to the excellent generalization performance, the feedforward-feedback control scheme can be easily extended to track different types of input waveforms with high accuracy. In the future, some pruning algorithms will be studied to reduce the sample size and speed up the computing performance.

\section{REFERENCES}

[1] H.-J. Shieh, F.-J. Lin, P.-K. Huang, and L.-T. Teng, "Adaptive displacement control with hysteresis modeling for piezoactuated positioning mechanism," IEEE Trans. Ind. Electron., vol. 53, no. 3, pp. 905-914, Jun. 2006.

[2] X. Chen, C.-Y. Su, Z. Li, and F. Yang, "Design of implementable adaptive control for micro/nano positioning system driven by piezoelectric actuator", IEEE Trans. Ind. Electron., vol. 63, no. 10, pp. 6471-6481, Oct. 2016.
[3] P. Ge and M. Jouaneh, "Tracking control of a piezoceramic actuator," IEEE Trans. Control Syst. Technol., vol. 4, no. 3, pp. 209-216, Apr. 1996.

[4] L.-S. Chen, J.-Y. Yen, J. H. Chen, F.-C. Kuo, M.-S. Chen, Y.-Y. Chen, and B.-I. Chung, "Precision tracking of a piezo-driven stage by charge feedback control," Precis. Eng., vol. 37, pp. 793-804, Oct. 2013.

[5] J. Minase, T.-F. Lu, B. Cazzolato, and S. Grainger, "A review, supported by experimental results, of voltage, charge and capacitor insertion method for driving piezoelectric actuators," Precis. Eng., vol. 34, no. 692-700, Oct. 2010.

[6] I. D. Mayergoyz, "Mathematical models of hysteresis," IEEE Trans. Magn., vol. 22, no. 5, pp. 603-608, Sep. 1986.

[7] P. Ge and M. Jouaneh, "Generalized Preisach model for hysteresis nonlinearity of piezoceramic actuator," Precis. Eng., vol. 20, no. 2, pp. 99-111, Mar. 1997.

[8] M. Ruderman, F. Hoffmann, and T. Bertram, "Modeling and identification of elastic robot joints with hysteresis and backlash," IEEE Trans. Ind. Electron., vol. 56, no. 10, pp. 3840-3847, Oct. 2009.

[9] C. Y. Su, Q. Wang, X. Chen, and S. Rakheja, "Adaptive variable structure control of class of nonlinear systems with unknown Prandtl-Ishlinskii hysteresis," IEEE Trans. Autom. Control, vol. 50, no. 12, pp. 2069-2074, Dec. 2005.

[10] M. A. Krasnoselskii and A. V. Pokrovskii, System with Hysteresis. New York, NY, USA: Springer, 1989.

[11] Y. Li, S. Tong, and T. Li, "Adaptive fuzzy output feedback control of uncertain nonlinear systems with unknown backlash-like hysteresis," Inf. Sci., vol. 198, no. 1, pp. 130-146, Sep. 2012.

[12] Y. Li, S. Tong, L. Liu, and G. Feng, "Adaptive output-feedback control design with prescribed performance for switched nonlinear systems," Automatica, vol. 80, pp. 225-231, Jun. 2017.

[13] R. B. Mrad and H. Hu, "A model for voltage-to-displacement dynamics in piezoceramic actuator subject to dynamic-voltage excitations," IEEE/ASME Trans. Mechatronics, vol. 7, no. 4, pp. 479-489, Dec. 2002.

[14] Y. Yu, Z. Xiao, N. G. Naganathan, and R. V. Dukkipati, "Dynamic Preisach modeling of hysteresis for the piezoceramic actuator system," Mech. Mach. Theory, vol. 37, no. 1, pp. 75-89, Jan. 2002.

[15] W. T. Ang, P. K. Khosla, and C. N. Riviere, "Feedforward controller with inverse rate-dependent model for piezoelectric actuators in trajectory-tracking applications," IEEE/ASME Trans. Mechatronics, vol. 12, no. 2, pp. 134-142, Apr. 2007.

[16] G.-Y. Gu, L.-M. Zhu, and C.-Y. Su, "Modeling and compensation of asymmetric hysteresis nonlinearity for piezoceramic actuators with a modified Prandtl-Ishlinskii model," IEEE Trans. Ind. Electron., vol. 61, no. 3, pp. 1583-1595, Mar. 2014.

[17] R. Xiong, X.-D. Liu, and Z.-L. Lai, "Heuristic modeling and inverse compensation of hysteresis in piezoelectric actuators based on time series similarity," J. Intell. Mater. Syst. Struct., vol. 27, no. 13, pp. 1814-1828, 2016.

[18] Z.-L. Lai, Z. Chen, X.-D. Liu, and Q.-H. Wu, "A novel similarity-based hysteresis empirical model for piezoceramic actuators," Sens. Actuators A: Phys., vol. 197, pp. 150-165, 2013.

[19] N. Dong, J. Gao, X. Liu, and X. Mao, "Study on the time-scale similarity of Bouc-Wen hysteresis model," in Proc. 2nd Int. Workshop Mater. Eng. Comput. Sci., Jinan, China, 2015, pp. 778-781.

[20] V. Vapnik, The Nature of Statistical Learning Theory. New York, NY, USA: Springer, 1995.

[21] F. Kaytez, M. C. Taplamacioglu, E. Cam, and F. Hardalac, "Forecasting electricity consumption: A comparison of regression analysis, neural networks and least squares support vector machine," Int. J. Electr. Power Energy Syst., vol. 67, pp. 431-438, May 2015.

[22] L. J. Cao and F. H. Tay, "Support vector machine with adaptive parameters in financial time series forecasting," IEEE Trans. Neural Netw., vol. 14, no. 6, pp. 1506-1518, Nov. 2003.

[23] R. Darnag, B. Minaoui, and M. Fakir, "QSAR models for prediction study of HIV protease inhibitors using support vector machines, neural networks and multiple linear regression," Arabian J. Chem., vol. 10, pp. S600-S608, Feb. 2017.

[24] Y. Ma, X. Zhang, M. Xu, and S. Xie, "Hybrid model based on Preisach and support vector machine for novel dual-stack piezoelectric actuator," Mech. Syst. Sig. Process., vol. 34, no. 1-2, pp. 156-172, Jan. 2013. 
[25] P.-K. Wong, Q. Xu, C.-M. Vong, and H.-C. Wong, "Rate-dependent hysteresis modeling and control of a piezostage using online support vector machine and relevance vector machine," IEEE Trans. Ind. Electron., vol. 59, no. 4, pp. 1988-2001, Apr. 2012.

[26] X. Mao, Y. Wang, X. Liu, and Y. Guo, "An adaptive weighted least square support vector regression for hysteresis in piezoelectric actuators," Sens. Actuators A: Phys., vol. 263, pp. 423-429, 2017.

[27] J. Yang, A. Bouzerdoum, S. Phung, "A training algorithm for sparse LS-SVM using compressive sampling," in Proc. Int. Conf. Acoustics, Speech, Signal Process. (ICASSP), 2010, pp. 2054-2057.

[28] W. Zhao, T. Tao, E. Zio, and W. Wang, "A novel hybrid method of parameters tuning in support vector regression for reliability prediction: Particle swarm optimization combined with analytical selection," IEEE Trans. Rel., vol. 65, no. 3, pp. 1393-1405, Feb. 2016.

[29] R. G. Gorjaei, R. Songolzadeh, M. Torkaman, M. Safari, and G. Zargar, "A novel PSO-LSSVM model for predicting liquid rate of two phase flow through wellhead chokes," J. Nat. Gas Sci. Eng., vol. 24, pp. 228-237, May 2015.

[30] W.-C. Hong, Y. Dong, L.-Y. Chen, and S.-Y. Wei, "SVR with hybrid chaotic genetic algorithms for tourism demand forecasting," Appl. Soft Comput., vol. 11, no. 2, pp. 1881-1890, Mar. 2011.

[31] J. Sun and X. Liu, "A novel APSO-aided maximum likelihood identification method for Hammerstein systems," Nonlinear Dyn., vol. 73, no. 1, pp. 449-462, Jul. 2013.

[32] Y. Ding, L. Cheng, W. Pedrycz, and K. Hao, "Global nonlinear kernel prediction for large data set with a particle swarm-optimized interval support vector regression," IEEE Trans. Neural Netw. Learn. Syst., vol. 26, no. 10, pp. 2521-2534, Oct. 2015.

[33] D. Habineza, M. Rakotondrabe, and Y. L. Gorrec, "Bouc-Wen modeling and feedforward control of multivariable hysteresis in piezoelectric systems: application to a 3-DoF piezotube scanner," IEEE Trans. Control Syst. Technol., vol. 23, no. 5, pp. 1797-1806, Sep. 2015.

[34] M. A. Janaideha and P. Krejčí, "Inverse rate-dependent Prandtl-Ishlinskii model for feedforward compensation of hysteresis in a piezomicropositioning actuator," IEEE/ASME Trans. Mechatronics, vol. 18, no. 5, pp. 1498-1507, Oct. 2013.

[35] Y. Qin, Y. Tian, D. Zhang, B. Shirinzadeh, and S. Fatikow, "A novel direct inverse modeling approach for hysteresis compensation of piezoelectric actuator in feedforward applications," IEEE/ASME Trans. Mechatronics, vol. 18, no. 3, pp. 981-989, Jun. 2013.

[36] R. Napoli and L. Piroddi, "Nonlinear active noise control with NARX models," IEEE Trans. Audio, Speech, Lang. Process., vol. 18, no. 2, pp. 286-295, Feb. 2010

[37] H. K. Sahoo, P. K. Dash, and N. P. Rath, "NARX model based nonlinear dynamic system identification using low complexity neural networks and robust $\mathrm{H}_{\infty}$ filter," Appl. Soft Comput., vol. 13, no. 7, pp. 3324-3334, Jul. 2013.

[38] H. Wang and G. Song, "Innovative NARX recurrent neural network model for ultra-thin shape memory alloy wire," Neurocomputing, vol. 134, no. 25, pp. 289-295, Jun. 2014.

[39] H. Asgari, X. Chen, M. Morini, M. Pinelli, R. Sainudiin, P. R. Spina, and Mauro Venturini, "NARX models for simulation of the start-up operation of a single-shaft gas turbine," Appl. Therm. Eng., vol. 93, no. 25, pp. 368-376, Jan. 2016.

[40] Y. Lv, F. Hong, T. Yang, and F. Fang, "A dynamic model for the bed temperature prediction of circulating fluidized bed boilers based on least squares support vector machine with real operational data," Energy, vol. 124, pp. 284-294, Apr. 2017. 